\title{
The Poisson's problem for the Laplacian with Robin boundary condition in non-smooth domains
}

Loredana Lanzani and Osvaldo Méndez

\begin{abstract}
Given a bounded Lipschitz domain $\Omega \subset \mathbb{R}^{n}, n \geq 3$, we prove that the Poisson's problem for the Laplacian with right-hand side in $L_{-t}^{p}(\Omega)$, Robin-type boundary datum in the Besov space $B_{p}^{1-1 / p-t, p}(\partial \Omega)$ and non-negative, non-everywhere vanishing Robin coefficient $b \in$ $L^{n-1}(\partial \Omega)$, is uniquely solvable in the class $L_{2-t}^{p}(\Omega)$ for $\left(t, \frac{1}{p}\right) \in \mathcal{V}_{\epsilon}$, where $\mathcal{V}_{\epsilon}(\epsilon \geq 0)$ is an open $(\Omega, b)$-dependent plane region and $\mathcal{V}_{0}$ is to be interpreted ad the common (optimal) solvability region for all Lipschitz domains. We prove a similar regularity result for the Poisson's problem for the 3-dimensional Lamé System with traction-type Robin boundary condition. All solutions are expressed as boundary layer potentials.
\end{abstract}

\section{Introduction}

In this article we use layer potentials to obtain optimal regularity results for the Poisson's problem for the Laplacian with Robin boundary condition and for the 3-dimensional Lamé system with traction-Robin boundary condition, on a bounded, non-smooth (Lipschitz) domain. Specifically, given a bounded Lipschitz domain $\Omega \subset \mathbb{R}^{n}$ and a non-negative, non-everywhere vanishing scalar function $b$, we study the Poisson's problem for the Laplacian with Robin boundary condition

$$
\left\{\begin{array}{l}
\Delta u=f \in L_{-t, 0}^{p}(\Omega) \quad \text { in } \Omega \\
\frac{\partial u}{\partial N}+b u=g \in B_{p}^{1-\frac{1}{p}-t, p}(\partial \Omega) \quad \text { on } \partial \Omega,
\end{array}\right.
$$

2000 Mathematics Subject Classification: 45E99, 47G10, 46E35.

Keywords: Non-smooth domains, Besov spaces, Triebel-Lizorkin spaces, boundary layer potentials, regularity of PDE's, Robin condition, Lamé system, Poisson's problem. 
and, for $n=3$, the Lamé System with traction-Robin boundary condition

$$
\left\{\begin{array}{l}
\mathcal{L} \mathbf{u}=\mathbf{f} \in L_{-t, 0}^{p}(\Omega) \quad \text { in } \Omega \\
\frac{\partial \mathbf{u}}{\partial \nu}+b \mathbf{u}=\mathbf{g} \in \mathbf{B}_{p}^{1-\frac{1}{p}-t, p}(\partial \Omega) \quad \text { on } \partial \Omega .
\end{array}\right.
$$

Here $L_{s, 0}^{p}(\Omega)$ stands for a suitable variant of the usual potential spaces defined via the Fourier transform (see Section 2 for the relevant definitions). For a three-dimensional vector field $\mathbf{u}$ defined in $\Omega$ and $\mu>0, \lambda>-\frac{2 \mu}{3}$, the differential operator in (1.2) is defined by

$$
\mathcal{L} \mathbf{u}:=\mu \Delta \mathbf{u}+(\lambda+\mu) \nabla \operatorname{div} \mathbf{u} .
$$

The boundary condition in (1.2) with $b=0$ has been extensively studied in the literature due to its importance in elasticity theory (see [4], [3], [17]). Our main results are Theorems 4.5 and 5.2 for problem (1.1) and Theorems 6.4 and 6.5 for the Lamé system (1.2).

We aim to expressing the solutions as the sum of a Newtonian potential (for Problem (1.2), elastic potential) and a single layer potential on the boundary. To this end, we solve first the homogeneous versions $(f=0)$ of (1.1) and (1.2) and show that the (unique) solution can be expressed in the form

$$
u(X)=\mathcal{S} \mathcal{T}^{-1}(g)(X),
$$

where $\mathcal{T}$ is an operator acting on an appropriate function space (Section 4) and $\mathcal{S}$ is the single layer potential on $\partial \Omega$ (see (1.7)). In both cases we first establish $L^{2}(\partial \Omega)$ and Hardy-Space type results for the invertibility of the solution operator $\mathcal{T}$. Then, via interpolation, we extend the invertibility of $\mathcal{T}$ to Besov spaces $B_{p}^{s, p}(\partial \Omega)$ for $p$ and $s$ ranging throughout an (optimal) region $\mathcal{U}_{\epsilon}$, where the positive number $\epsilon$ depends on the Robin coefficient $b$ and the Lipschitz character of $\Omega$. The solution for the non-homogeneous problems is then obtained by adding the Newtonian potential of $f$ to (1.4), see (5.3).

The paper is structured as follows: In Section 1, we briefly present the notation and terminology to be used throughout the article; in Section 2 we introduce the function spaces needed for the proper formulation of (1.1) and (1.2). In Section 3 we investigate the Fredholm index of the solution operator $\mathcal{T}$ on various function spaces; Section 4 is devoted to the study of invertibility of the boundary operator associated with (1.1) on suitable function spaces; the solvability of (1.1) is presented in Section 5. The invertibility and solvability results for Problem (1.2) are given in Section 6 . 
Given a Lipschitz domain $\Omega \subset \mathbb{R}^{n}$ with boundary $\partial \Omega,(\gamma(P))_{P \in \partial \Omega}$ denotes a fixed family of two-sided non-tangential cones (see [23]). As usual, $+\gamma(P) \subseteq \Omega$ and $-\gamma(P) \subseteq \mathbb{R}^{n} \backslash \Omega$ symbolize the interior and exterior part of $\gamma(P)$ respectively. For $u: \Omega \rightarrow \mathbb{C}, P \in \partial \Omega$, let

$$
M(u)(P)=\sup \{|u(X)|, X \in \gamma(P)\}
$$

denote the $\left((\gamma(P))_{P \in \partial \Omega}\right.$-dependent) non-tangential maximal function. As is customary, $X$ stands for a generic point in $\mathbb{R}^{n} \backslash \Omega$, while the notation $P$ or $Q$ is reserved for points on $\partial \Omega$; surface measure will be written as $d \sigma$. The $(d \sigma$-a.e defined) exterior unit normal vector at a point $P \in \partial \Omega$ will be denoted by $N(P)$.

Let $\Gamma$ be the fundamental solution of the Laplacian, i.e.,

$$
\Gamma(X)=\frac{1}{(2-n) \omega_{n}|X|^{n-2}}, X \in \mathbb{R}^{n} \backslash\{0\},
$$

where $\omega_{n}(n \geq 3)$ stands for the surface measure of the unit ball in $\mathbb{R}^{n}$. Let $\mathcal{L}(\partial \Omega)$ denote the space of Lipschitz functions on $\partial \Omega$ and let $\mathcal{L}^{\prime}(\partial \Omega)$ stand for its dual. In preparation for Section 4 , we define the single layer potential with density $\Lambda \in \mathcal{L}^{\prime}(\partial \Omega)$ as

$$
\mathcal{S}(\Lambda)(X)=\langle\Lambda, \Gamma(X-\cdot)\rangle, X \in \mathbb{R}^{n} \backslash \partial \Omega .
$$

The boundary trace is written as

$$
S(\Lambda)(P)=\langle\Lambda, \Gamma(P-\cdot)\rangle, P \in \partial \Omega .
$$

In particular, if $f \in L^{1}(\partial \Omega)$,

$$
\mathcal{S}(f)(X)=\int_{\partial \Omega} f(Q) \Gamma(X-Q) d \sigma(Q) .
$$

The double layer potential with density $f: \partial \Omega \rightarrow \mathbb{C}$ is defined as

$$
\mathcal{D}(f)(X)=\int_{\partial \Omega} f(Q) \frac{\partial}{\partial N(Q)} \Gamma(X-\cdot) d \sigma(Q) ;
$$

these potentials are well defined and harmonic in $X \in \mathbb{R}^{n} \backslash \partial \Omega$. As a consequence of the $L^{2}$-boundedness of the Cauchy integral operator on Lipschitz curves $([1])$, the singular integral operators

$$
K f(P)=p \cdot v \cdot \int_{\partial \Omega} f(Q) \frac{\partial}{\partial N(Q)} \Gamma(P-\cdot) d \sigma(Q)
$$

and its formal transpose

$$
K^{*} f(P)=p \cdot v \cdot \int_{\partial \Omega} f(Q) \frac{\partial}{\partial N(P)} \Gamma(P-\cdot) d \sigma(Q)
$$

are bounded on $L^{p}(\partial \Omega)$ for $1<p<\infty$. Furthermore, $K^{*}$ is bounded on $H^{p}(\partial \Omega)$ (the atomic Hardy space, defined on $\partial \Omega$, see $\left.([2])\right)$ for $\frac{n-1}{n}<p \leq 1$. 
We refer the reader to Section 2 for the definition of the Besov spaces $B_{p}^{s, p}$ on $\partial \Omega$; meanwhile we state the trace properties of the layer potentials: for a detailed discussion, see [23].

For $1<p<\infty$ and $f \in L^{p}(\partial \Omega)$, the following trace formulae hold:

$$
\lim _{ \pm \gamma(P) \ni X \rightarrow P} \mathcal{D} f(X)= \pm \frac{1}{2} f(P)+K f(P) d \sigma-\text { a.e. } P
$$

and

$$
\begin{aligned}
\frac{\partial}{\partial N} \mathcal{S}(f)(P) & =\lim _{ \pm \gamma(P) \ni X \rightarrow P}\langle\nabla \mathcal{S}(X), N(P)\rangle \\
& =\mp \frac{1}{2} f(P)+K^{*} f(P) d \sigma-\text { a.e. } P,
\end{aligned}
$$

where $\langle a, b\rangle$ denotes the Euclidean scalar product in $\mathbb{R}^{n}$. For $1 \leq p<\infty$, $0<s<1$ and $f \in B_{p}^{s, p}(\partial \Omega)$, (1.13) takes up the form

$$
\operatorname{Tr} \mathcal{D} f= \pm \frac{1}{2} f(P)+K f(P),
$$

where Tr stands for the trace operator (see [12]). For $f \in B_{p}^{-s, p}(\partial \Omega$ ), the "interior" statement in (1.14) is to be understood in the following weak sense (see [8] for the details): for arbitrary $\phi \in B_{q}^{s, q}(\partial \Omega)\left(q=\frac{p}{p-1}\right)$ extended to $\Omega$ as a function in $L_{q}^{s+1 / q, q}(\Omega)$, the equality

$$
\langle\nabla \mathcal{S}, \nabla \phi\rangle=\left\langle\left(-\frac{1}{2} I+K^{*}\right) f, \phi\right\rangle
$$

holds.

\section{Function spaces and potentials}

In this section we introduce the various spaces that naturally arise in the study of the regularity problems under consideration here. Once again $\Omega \subset$ $\mathbb{R}^{n}, n \geq 3$, will stand for a bounded, Lipschitz domain with connected boundary $\partial \Omega$. For $0<p, q \leq \infty$, let $A_{p}^{s, q}\left(\mathbb{R}^{n}\right)$ denote either the Besov space $B_{p}^{s, q}\left(\mathbb{R}^{n}\right)$ or the Triebel-Lizorkin space $F_{p}^{s, q}\left(\mathbb{R}^{n}\right)$ (e.g [21] for the definition and a complete survey on these spaces). For $s \geq 0$ the corresponding spaces $A_{p}^{s, q}(\partial \Omega)$ are defined by localization, whereas for $t>0$ and $1<p, q \leq \infty$, we define

$$
A_{p}^{-t, q}(\partial \Omega)=\left(A_{p^{\prime}}^{t, q^{\prime}}(\partial \Omega)\right)^{*}
$$

where $p^{\prime}$ and $q^{\prime}$ stand for the Hölder-conjugate exponents of $p$ and $q$ respectively. The spaces $A_{p}^{s, q}(\Omega)$ are defined as spaces of distributions (in $\Omega$ ) 
obtained by restriction to $\Omega$ of distributions in $A_{p}^{s, q}$; more precisely, for $s \in \mathbb{R}$ and $p, q \in(0, \infty]$, we let

$$
A_{p}^{s, q}(\Omega)=\left\{f \in \mathcal{D}^{\prime}(\Omega): f=\left.F\right|_{\Omega}, F \in A_{p}^{s, q}\left(\mathbb{R}^{n}\right)\right\} .
$$

These spaces are Banach when endowed with the quotient norm

$$
\|f\|_{A_{p}^{s, q}(\Omega)}=\inf \left\{\|F\|_{A_{p}^{s, q}\left(\mathbb{R}^{n}\right)},\left.F\right|_{\Omega}=f\right\} .
$$

Furthermore, for $s, p$ and $q$ as above, we let

$$
\dot{A}_{p}^{s, q}(\Omega)=\left\{f \in \mathcal{D}^{\prime}\left(\mathbb{R}^{n}\right): \operatorname{supp} f \subseteq \Omega\right\} .
$$

For $s \in \mathbb{R}, 1<p, q<\infty$, the following equalities hold for the dual spaces:

$$
\left(\dot{A}_{p}^{s, q}(\Omega)\right)^{*}=A_{p^{\prime}}^{s, q^{\prime}}(\Omega)
$$

and

$$
\left(A_{p}^{s, q}(\Omega)\right)^{*}=\left(\dot{A}_{p^{\prime}}^{s, q^{\prime}}(\Omega)\right) .
$$

See [11] for a proof of (2.2). The identity (2.3) was proved in [8] for $p=q$; a slight modification of the arguments presented there makes both proofs work also for $p \neq q$. We recall that for $p \in(0, \infty]$, the embedding

$$
F_{p}^{1,2}(\mathbf{D}) \rightarrow F_{p}^{0,2}(\mathbf{D})
$$

is well defined and compact for any smooth, bounded domain D (see [5]). Based on this observation and using a customary localization and duality argument it is not hard to prove that for a bounded Lipschitz domain the embedding

$$
i: F_{p}^{1,2}(\partial \Omega) \rightarrow F_{p}^{0,2}(\partial \Omega)
$$

is well defined and compact.

For real $s$, let $L_{s}^{p}\left(\mathbb{R}^{n}\right)$ denote the potential spaces defined via the Fourier transform (see [11]), i.e.,

$$
L_{s}^{p}\left(\mathbb{R}^{n}\right)=\left\{(I-\Delta)^{-\frac{s}{2}}(g): g \in L^{p}\left(\mathbb{R}^{n}\right)\right\},
$$

with

$$
\|f\|_{L_{s}^{p}}=\left\|(I-\Delta)^{\frac{s}{2}}(f)\right\|_{L^{p}} .
$$


It is well known (see [21]) that the Triebel-Lizorkin scale $\left(F_{p}^{s, 2}\right)_{s, p}$ contains both $L_{s}^{p}$ (which coincide with the classic Sobolev spaces when $\mathrm{s}$ is a nonnegative integer) and the atomic Hardy spaces $H^{p}, 0<p \leq 1$. More precisely, for $s \in \mathbb{R}$ and $1<p<\infty$, we have

$$
F_{p}^{s, 2}\left(\mathbb{R}^{n}\right)=L_{s}^{p}\left(\mathbb{R}^{n}\right),
$$

whereas, for $0<p \leq 1$,

$$
F_{p}^{0,2}\left(\mathbb{R}^{n}\right)=H_{i n}^{p}\left(\mathbb{R}^{n}\right),
$$

where the right hand side stands for the inhomogeneous spaces (no vanishing moment condition is required for the corresponding atoms (see also [9])). In the sequel, we will freely use (2.5) and (2.6). Occasionally, we will write $L_{s, 0}^{p}(\Omega)$ instead of $\dot{F}_{p}^{s, 2}(\Omega)$ (see $(2.1)$ ). In closing this section we recall a few results (see [8, Lemma 9.1] and preceding Remark) that will be needed in Section 5 .

Lemma 2.1 For $0<s, 1<p<\infty$ and $k=1,2, \ldots, n$ the partial derivative operator

$$
\partial_{k}: L_{s}^{p}(\Omega) \rightarrow L_{s-1}^{p}(\Omega)
$$

is well defined and bounded.

Lemma 2.2 If $0<s<1,1<q<\infty, \frac{1}{p}+\frac{1}{q}=1$ and $-s+\frac{1}{q} \leq 0$, then

$$
L_{-s+\frac{1}{q}}^{q}(\Omega)=\left(L_{s-1+\frac{1}{p}}^{p}(\Omega)\right)^{*},
$$

whereas if $-s+\frac{1}{q} \geq 0$, we have

$$
L_{s-1+\frac{1}{p}}^{p}(\Omega)=\left(L_{-s+\frac{1}{q}}^{q}(\Omega)\right)^{*} .
$$

Finally, with the same notation used in Lemma 2.2, given $\frac{1}{q}<t<1+\frac{1}{q}$ and an $n$-dimensional vector field $F \in L_{1-t}^{p}(\Omega)\left(\frac{1}{p}+\frac{1}{q}=1\right)$ whose divergence has a continuous extension to $L_{t}^{q}(\Omega)$ (i.e., if $\operatorname{div} F \in L_{-t, 0}^{p}(\Omega)$ ), we identify the normal component $F \cdot N$ of $F$ with the linear functional in $B_{p}^{1-1 / p-t, p}(\partial \Omega)$ (which depends on the aforementioned extension), defined via:

$$
\langle F \cdot N, \phi\rangle=\langle\operatorname{div} F, \tilde{\phi}\rangle+\langle F, \nabla \tilde{\phi}\rangle
$$

for every $\phi \in B_{t-1 / q}^{q}(\partial \Omega)$ extended to $\tilde{\phi} \in L_{t}^{q}(\Omega)$. Notice that the normal component does not depend on the particular extension $\tilde{\phi}$. Furthermore,

$$
\|F \cdot N\|_{B_{1-\frac{1}{p}-t}^{p}(\partial \Omega)} \leq C(t, p, \Omega)\left(\|F\|_{L_{1-t}^{p}(\Omega)}+\|\operatorname{div} F\|_{L_{t}^{q}(\Omega)}\right) .
$$




\subsection{The Newtonian Potential}

We now define the Newtonian potential and investigate its main features as an operator on the Sobolev and Besov scales. Let $n \geq 3, \omega_{n}$ and $\Gamma$ be defined by (1.6). Consider the restriction operator $\mathcal{R}_{\Omega}$ (from $\mathbb{R}^{n}$ to $\Omega$ ) acting on distributions; let $\mathcal{E}^{\prime}(\Omega)$ stand for the space of compactly supported distributions in $\Omega$, and let $\mathcal{N}$ denote the convolution operator

$$
\mathcal{N}: \mathcal{E}^{\prime} \rightarrow \mathcal{D}^{\prime}
$$

defined by

$$
\mathcal{N}(\Lambda)=\Gamma * \Lambda
$$

We define the Newtonian potential on $\Omega$,

$$
\mathcal{N}_{\Omega}: \mathcal{E}^{\prime}(\Omega) \rightarrow \mathcal{D}^{\prime}(\Omega)
$$

as

$$
\mathcal{N}_{\Omega}(f)=\mathcal{R}_{\Omega} \mathcal{N}(\tilde{f})
$$

where $\tilde{f}$ denotes a compactly supported distribution in $\mathbb{R}^{n}$, which is defined via the identity

$$
\langle\tilde{f}, \phi\rangle=\left\langle f, \mathcal{R}_{\Omega}(\phi)\right\rangle .
$$

The Sobolev-Besov regularity of $\mathcal{N}_{\Omega}$, was studied in [8]:

Theorem 2.3 Let $1<p, q<\infty, \frac{1}{p}+\frac{1}{q}=1$ and $1 \leq s \leq 3$. Then the Newtonian potential defined by (2.9) is bounded as a linear operator

$$
\mathcal{N}_{\Omega}: L_{-s, 0}^{p}(\Omega) \rightarrow L_{2-s}^{p}(\Omega)
$$

Moreover, if $1<s<3, \mathcal{N}_{\Omega}$ is bounded as an operator

$$
\mathcal{N}_{\Omega}: B_{-s, 0}^{p}(\Omega) \rightarrow B_{2-s}^{p}(\Omega) .
$$

For a distribution $f \in L_{\frac{1}{p}-s-1,0}^{p}(\Omega)$ and $-1 \leq s-\frac{1}{p} \leq 2$, the vector field

$$
\nabla \mathcal{N}_{\Omega}(f) \in L_{-s+\frac{1}{p}}^{p}(\Omega)
$$

has normal component (denoted $\frac{\partial \mathcal{N}_{\Omega}(f)}{\partial N}$ ) in the space $B_{-s}^{q}(\partial \Omega)$.

Proof. See Proposition 2.1 and 9.7 in [8]. 


\subsection{Mapping properties of the boundary layer}

We conclude this Section by recalling the following mapping property of the single layer potential on the scale $B_{p}^{-s, p}(\partial \Omega)$ (with $0<s<1$ ). See [8] for further details.

Theorem 2.4 For $0<s<1$ and $1<p<\infty$, the single layer potential

$$
\mathcal{S}: B_{p}^{-s, p}(\partial \Omega) \rightarrow B_{p}^{1-s+\frac{1}{p}, p}(\Omega) \cap L_{1-s+\frac{1}{p}}^{p}(\Omega)
$$

is a bounded linear operator. Here, the intersection is endowed with the norm

$$
\|f\|_{B_{p}^{1-s+\frac{1}{p}, p}(\Omega) \cap L_{1-s+\frac{1}{p}}^{p}(\Omega)}=\max \left\{\|f\|_{B_{p}^{1-s+\frac{1}{p}, p}(\Omega)},\|f\|_{L_{1-s+\frac{1}{p}}^{p}(\Omega)}\right\} .
$$

\section{Fredholm analysis of the solution operator}

In this section we investigate the Besov-space regularity and the invertibility of the operator that gives the solution of the Poisson's problem (1.1) namely the operator $\mathcal{T}$ in (1.4). More precisely, we define $\mathcal{T}$ as (see (1.8) and [14]):

$$
\mathcal{T}=-\frac{1}{2} I+K^{*}+b S
$$

and we show that under certain conditions on $s$ and $p$ (involving the Lipschitz character of $\Omega$ ), $\mathcal{T}$ is invertible on the Besov scale $B_{p}^{-s, p}(\partial \Omega)$ (Theorem 4.5). To this end, we show that $\mathcal{T}$ has vanishing Fredholm index on appropriate Besov spaces (indeed, $\mathcal{T}$ is a compact perturbation of the solution operator for the Neumann problem). We start with a Lemma that plays a central role, both in this Section and in Section 5, where it is crucial to justify the weak formulation of the Robin boundary condition.

Lemma 3.1 Let $\Omega \subset \mathbb{R}^{n}$ denote a bounded Lipschitz domain with connected boundary. Assume that $b \in L^{n-1}(\partial \Omega), 1<q<\infty, n \geq 3$ and $0<s<1$. Let $T_{b}$ denote pointwise multiplication times $b$, namely

$$
T_{b}(\psi)=\psi b .
$$

Then $T_{b}$ is bounded as an operator

$$
T_{b}: B_{q}^{1-s, q}(\partial \Omega) \rightarrow B_{q}^{-s, q}(\partial \Omega) .
$$

Moreover, for $q<n-1, T_{b}$ is bounded as an operator

$$
T_{b}: L_{1}^{q}(\partial \Omega) \rightarrow L^{q}(\partial \Omega) .
$$


Proof. For $q<n-1$, assertion (3.4) follows from the Sobolev's embedding Theorem: for $\psi \in L_{1}^{q}(\partial \Omega)$, we have

$$
\begin{aligned}
\|b \psi\|_{L^{q}(\partial \Omega)} & \leq\|b\|_{L^{n-1}(\partial \Omega)}\|\psi\|_{L^{\frac{q(n-1)}{n-1-q}(\partial \Omega)}} \\
& \leq\|b\|_{L^{n-1}(\partial \Omega)}\|\psi\|_{L_{1}^{q}(\partial \Omega)} .
\end{aligned}
$$

We next claim that for $n \geq 3, T_{b}$ maps $B_{1}^{1-s, 1}(\partial \Omega)$ boundedly into $B_{1}^{-s, 1}(\partial \Omega)$. To see this, select $s=(n-1)\left(\frac{1}{p}-1\right)$ and let $a$ denote an $L^{t}-F_{p}^{1,2}$-atom for $1<t<n-1$, so that there exists a ball $B(r)$ of radius $r>0$, with

- $\operatorname{supp} a \subseteq B(r) \cap \partial \Omega$,

- $\|a\|_{F_{t}^{1,2}(\partial \Omega)} \leq r^{(n-1)\left(\frac{1}{t}-\frac{1}{p}\right)}$.

On account of well-known embedding theorems, we have that

$$
F_{t}^{1,2}(\partial \Omega) \subseteq L^{\frac{t(n-1)}{n-1-t}}(\partial \Omega)
$$

whence

$$
\|b a\|_{F_{t}^{0,2}(\partial \Omega)} \leq\|b\|_{L^{n-1}(\partial \Omega)}\|a\|_{F_{t}^{1,2}(\partial \Omega)} \leq c\|b\|_{L^{n-1}(\partial \Omega)} r^{(n-1)\left(\frac{1}{t}-\frac{1}{p}\right)},
$$

where $c$ is a positive constant independent on $a$.

Therefore, $b a$ is an $L^{t}-F_{p}^{0,2}(\partial \Omega)$-atom (see [9]) and it follows that $T_{b}$ is bounded as an operator

$$
T_{b}: F_{p}^{1,2}(\partial \Omega) \rightarrow F_{p}^{0,2}(\partial \Omega)
$$

with norm bounded by a constant times $\|b\|_{L^{n-1}(\partial \Omega)}$ (here that $F_{p}^{s, q}(\partial \Omega$ ) denotes the Triebel-Lizorkin space, see Section 2). Before completing the proof, we recall some functional-analytic tools from [17]. For a given quasiBanach space $X$, let $\hat{X}$ denote the Banach envelope of $X$, i.e. $\hat{X}$ is the "minimal" Banach space that contains $X$ (see [17] for the precise definition of the "hat" operation). Any continuous linear operator $T$ on $X$ has a natural linear and bounded extension $\hat{T}$ to $\hat{X}$. We recall that the hat operator preserves isomorphisms and compactness. More precisely,

$$
\|\hat{T}\|_{\mathcal{B}(\hat{X}, \hat{X})} \leq\|T\|_{\mathcal{B}(X, X)}
$$

and if $T$ is an isomorphism (or is compact) on $X$, so is $\hat{T}$ on $\hat{X}$. Moreover, it was proved in [17] that the following identifications hold for $0<p<1$ :

$$
\hat{F}_{p}^{0,2}(\partial \Omega)=B_{1}^{-(n-1)\left(\frac{1}{p}-1\right), 1}(\partial \Omega)
$$

and

$$
\hat{F}_{p}^{1,2}(\partial \Omega)=B_{1}^{1-(n-1)\left(\frac{1}{p}-1\right), 1}(\partial \Omega)
$$


Therefore, it follows from (3.5) that the "hat" extension of $T_{b}$, which, as it is easily verified, is still multiplication times $b$, maps $\hat{F}_{p}^{1,2}(\partial \Omega)=B_{1}^{1-s, 1}(\partial \Omega)$ boundedly into $\hat{F}_{p}^{0,2}(\partial \Omega)=B_{1}^{-s, 1}(\partial \Omega)$. It is now easily verified that $T_{b}$ is self-adjoint. Thus, $T_{b}$ is bounded as an operator

$$
T_{b}: B_{\infty}^{s, \infty}(\partial \Omega) \rightarrow B_{\infty}^{s-1, \infty}(\partial \Omega) .
$$

Standard interpolation arguments now yield the rest of the proof. We remark that the norm of $T_{b}$ as defined above satisfies the bound

$$
\left\|T_{b}\right\|_{\mathcal{B}\left(B_{p}^{1-s, p}(\partial \Omega), B_{p}^{-s, p}(\partial \Omega)\right)} \leq C\|b\|_{L^{n-1}(\partial \Omega)}
$$

for some positive constant $C=C(\Omega, p, s)$.

Recall now that for $0<s<1$ and $1<p<\infty$, the embedding

$$
B_{p}^{s, p}(\partial \Omega) \hookrightarrow B_{p}^{s-1, p}(\partial \Omega)
$$

is compact (see [5]). Next, on account of Lemma 3.1 and results in [23] we claim that for $0<s<1$ and $1<p<\infty$, the operator

$$
T_{b} \circ S: B_{p}^{s-1, p}(\partial \Omega) \rightarrow B_{p}^{s-1, p}(\partial \Omega)
$$

is also compact. Indeed, for smooth $b$ it is clear that $b S$ maps $B_{p}^{s-1, p}(\partial \Omega)$ into $B_{p}^{s, p}(\partial \Omega)$ (recall that $S$ is a bounded operator from $B_{p}^{s-1, p}(\partial \Omega)$ into $B_{p}^{s, p}(\partial \Omega)$ for $0<s<1$, see [8]); this observation and the bound (3.7) allow us to use a standard approximation argument to complete the proof of the claim. We now compute the Fredholm index of the operator $\mathcal{T}$ (see 3.1) on the Besov scale $B_{p}^{s-1, p}(\partial \Omega)$ for $0<s<1$. On account of $(3.9), \mathcal{T}$ has the same Fredholm index as the operator $-\frac{1}{2} I+K^{*}$. The latter is studied in the following Lemma.

Lemma 3.2 There exists $0<\epsilon=\epsilon(\Omega)$ depending on the Lipschitz character of $\Omega$ such that the operator $R=\frac{1}{2} I-K$ is Fredholm and has index 0 when acting on the following spaces:

(i) $L^{2}(\partial \Omega)$

(ii) $L_{1}^{2}(\partial \Omega)$

(iii) $B_{1}^{1-s, 1}(\partial \Omega)$ for $0<s<\epsilon$.

Also, for $s$ as in (iii), $R^{*}$ is Fredholm and has index 0 as an operator acting on $B_{\infty}^{s-1, \infty}(\partial \Omega)$, and closed range and index 0 as an operator on $F_{1}^{0,2}(\partial \Omega)$. 
Proof. The proofs of $(i)$ and $(i i)$ are essentially contained in [23], whereas (iii) was established in [2]: for the sake of completeness we sketch these proofs here. Let $\tilde{A}_{p}^{s, q}(\partial \Omega)$ stand for the elements in (the space of distributions) $A_{p}^{s, q}(\partial \Omega)$ which vanish on constants. It is well known (see [23]) that as an operator on $L^{2}(\partial \Omega), R^{*}$ has closed range; moreover, range $\left(R^{*}\right)=$ $\tilde{L}^{2}(\partial \Omega)=\tilde{F}_{p}^{0,2}(\partial \Omega)$, and $\operatorname{ker}\left(R^{*}\right)=\left\langle f_{0}\right\rangle$, where $\int_{\partial \Omega} f_{0}=1$ and $S\left(f_{0}\right)$ is a non-zero constant. Thus, the index of $R$ on $L^{2}(\partial \Omega)$ is 0 and, furthermore, $R^{*}$ is invertible as an operator on $\tilde{L}^{2}(\partial \Omega)$. The following topological decomposition is easily verified:

$$
L^{2}(\partial \Omega)=\left\langle f_{0}\right\rangle \oplus \tilde{L}^{2}(\partial \Omega)
$$

whence

$$
L^{2}(\partial \Omega)={ }^{\perp}\left(\left\langle f_{0}\right\rangle\right) \oplus\langle 1\rangle=\operatorname{range}(R) \oplus\langle 1\rangle .
$$

Now $R$ is bounded as an operator acting on $L_{1}^{2}(\partial \Omega)$, (see [23]). Let $g \in$ $L^{2}(\partial \Omega)$ and $R(g)=f \in L_{1}^{2}(\partial \Omega)$. Write

$$
u(X)=S\left(R^{*}\right)^{-1}\left(S^{-1}(f)-\left(\int_{\partial \Omega} S^{-1} f d \sigma\right) f_{0}\right)(X) .
$$

Integration by parts in $\Omega$ yields

$$
u(X)=\mathcal{D}\left(\left.u\right|_{\partial \Omega}\right)(X)-\mathcal{S}\left(\frac{\partial u}{\partial N}\right)(X) .
$$

In particular, there exist a constant $c$ and a function $h \in L_{1}^{2}(\partial \Omega)$ such that

$$
R(h)=c+f=c+R(g),
$$

from which it follows that $c=0$ and $g \in L_{1}^{2}(\partial \Omega)$, hence the closedness assertion in (ii). We also note that the above argument shows that the range of $R$ as an operator on $L_{1}^{2}(\partial \Omega)$ has codimension 1 (see( 3.10$)$ ), from which we obtain

$$
L_{1}^{2}(\partial \Omega)=\langle 1\rangle \oplus \operatorname{range}(R)
$$

The kernel of $R$ on $L_{1}^{2}(\partial \Omega)$ is the subspace generated by the constant 1 . Thus, the Fredholm index of $R$ on $L_{1}^{2}(\partial \Omega)$ is 0 , as claimed. We note in passing that these observations imply that the range of $R^{*}$ as an operator on $L_{-1}^{2}(\partial \Omega)$ is closed and equal to $\tilde{L}_{1}^{2}(\partial \Omega)$, whence, as an operator on $L_{-1}^{2}(\partial \Omega)$, $\operatorname{ker}\left(R^{*}\right)=\left\langle f_{0}\right\rangle$.

Finally, it was proved in [8] that for $0<s<\epsilon=\epsilon(\Omega)$, the operator

$$
R: B_{1}^{1-s, 1}(\partial \Omega) \rightarrow B_{1}^{1-s, 1}(\partial \Omega)
$$

is bounded and has closed range in $B_{1}^{1-s, 1}(\partial \Omega)$, and that the single layer potential operator, $S$ (which is self-adjoint) is an isomorphism from $B_{1}^{-s, 1}(\partial \Omega)$ onto $B_{1}^{1-s, 1}(\partial \Omega)$, hence also from $B_{\infty}^{s-1, \infty}(\partial \Omega)$ onto $B_{\infty}^{s, \infty}(\partial \Omega)$. 
Since

$$
L_{1}^{2}(\partial \Omega)=B_{2}^{1,2}(\partial \Omega) \subseteq B_{1}^{1-s, 1}(\partial \Omega),
$$

the unique function $g_{0} \in B_{\infty}^{s-1, \infty}(\partial \Omega)$ with $S\left(g_{0}\right)=1$ is a non-zero multiple of the generator of the kernel of $R^{*}$ in $L_{-1}^{2}(\partial \Omega)$. Thus, when $R^{*}$ is considered as an operator on $B_{\infty}^{s-1, \infty}(\partial \Omega)$, we have

$$
\operatorname{ker}\left(R^{*}\right)=\left\langle f_{0}\right\rangle
$$

It is easy to see that the range of $R^{*}$ on the above space also coincides with $\tilde{B}_{\infty}^{s-1, \infty}(\partial \Omega)$ (since $R^{*}$ is an isomorphism there). We now proceed to prove the last statement in Lemma 3.2. The closedness of the range of $R^{*}$ as an operator on $F_{1}^{0,2}(\partial \Omega)$ follows from [2]; indeed, it is shown there that the range of $R^{*}$ coincides with the space of functions in $F_{1}^{0,2}(\partial \Omega)$ which have vanishing integral. Recall that $L^{2}(\partial \Omega)=F_{2}^{0,2}(\partial \Omega) \subseteq F_{1}^{0,2}(\partial \Omega)$ (see [19]). Selecting $f \in F_{1}^{0,2}(\partial \Omega)$ with $R^{*}(f)=0$, integration by parts in $\Omega$ (which is legitimate since $M(f) \in L^{1}(\partial \Omega)$, see(1.5)) in conjunction with the fact that $S: F_{1}^{0,2}(\partial \Omega) \rightarrow F_{1}^{1,2}(\partial \Omega)$ is an isomorphism (see [2] and [17]) easily yields that $f$ is a multiple of the generator of $\operatorname{ker}\left(R^{*}\right)$ and from this, it follows immediately that the index of $R^{*}$ is 0 .

\section{Invertibility of the solution operator}

Throughout this section we fix $b \in L^{n-1}(\partial \Omega)$. The solution operator to the Poisson's problem (1.1) (with $f=0$ ) was defined in (3.1).

Lemma 4.1 If $b \geq 0, b>0$ on a set of positive Surface measure and $n \geq 3$, the operator $\mathcal{T}$ is invertible on $F_{1}^{0,2}(\partial \Omega)$.

Proof. Let $f \in F_{1}^{0,2}(\partial \Omega)$ with $\mathcal{T}(f)=0$. Since $M(\nabla S(f)) \in L^{1}(\partial \Omega)$ (see [2]), we may integrate by parts and obtain

$$
\int_{\Omega}|\nabla \mathcal{S} f(X)|^{2} d x=-\int_{\partial \Omega} b(P) S(f)^{2}(P) d \sigma(P),
$$

from which it can be easily seen that $\mathcal{T}$ is injective on $F_{1}^{0,2}(\partial \Omega)$ (see [14]). Next, we show that

$$
b S: F_{1}^{0,2}(\partial \Omega) \rightarrow F_{1}^{0,2}(\partial \Omega)
$$

is compact. To this end we recall the atomic decomposition of the above spaces: we refer the reader to [9] and [17] for further details. Let $A$ be an $L_{1}^{p}-F_{1}^{1,2}(\partial \Omega)$ atom , i.e., there exists a number $r, 0<r<\epsilon(\partial \Omega)$,

- $\operatorname{supp} A \subseteq B(r) \cap \partial \Omega$

- $\|A\|_{F_{p}^{1,2}(\partial \Omega)} \leq c\left(r^{n-1}\right)^{\frac{1}{p}-1}$. 
Choosing $1<p<n-1$, Hölder's inequality and the Sobolev Embedding Theorem grant

$$
\begin{aligned}
\|b A\|_{L^{p}(\partial \Omega)} & \leq\|b\|_{L^{n-1}(\partial \Omega)}\|A\|_{L^{\frac{(n-1) p}{n-1-p}(\partial \Omega)}} \\
& \leq\|b\|_{L^{n-1}(\partial \Omega)}\|A\|_{L_{1}^{p}(\partial \Omega)} \leq\|b\|_{L^{n-1}(\partial \Omega)} r^{(n-1)\left(\frac{1}{p}-1\right)} .
\end{aligned}
$$

Thus $b A$ is a $p$-atom for $F_{1}^{0,2}(\partial \Omega$ ) (see [9]) and the boundedness of the operator in (4.2) follows at once. Since the operator (4.2) can be approximated (in the operator norm) by $b_{j} S$, for smooth (Lipschitz) functions $b_{j}$, the compactness statement will follow once it has been established for Lipschitz $b$. Indeed, multiplication by a smooth $b$ defines a bounded operator on $F_{1}^{1,2}(\partial \Omega)$, thus for such a $b$ the compactness result is a direct consequence of the compact embedding (2.4). This, in conjunction with (iii) in Lemma 3.2 and the above injectivity result, yield the invertibility of $\mathcal{T}$ on $F_{1}^{0,2}(\partial \Omega)$, as claimed.

Lemma 4.2 The operator $\mathcal{T}$ has index 0 on $B_{2}^{-1,2}(\partial \Omega)$ for $n>3$. For $n=3, \mathcal{T}$ has index 0 on $B_{2}^{-s, 2}(\partial \Omega)$ for $0<s<1$, and index 0 on $B_{2}^{-1,2}(\partial \Omega)$ if we assume $b \in L^{r}(\partial \Omega)$ for some $r>2$.

Proof. On account of $(i i)$ in Lemma 3.2, the claim will follow once we show that $b S$ is compact as an operator on $B_{2}^{-1,2}(\partial \Omega)=L_{-1}^{2}(\partial \Omega)$. To this end we first consider the case $n>3$ and show the bound

$$
\|b S\|_{\mathcal{B}\left(L_{-1}^{2}(\partial \Omega), L_{-1}^{2}(\partial \Omega)\right)} \leq\|b\|_{L^{n-1}(\partial \Omega)}\|S\|_{\mathcal{B}\left(L_{-1}^{2}(\partial \Omega), L_{-1}^{2}(\partial \Omega)\right)},
$$

where $b \in \mathcal{L}(\partial \Omega)$. To prove (4.3), note that for $\Lambda \in L_{-1}^{2}(\partial \Omega)$ we have,

$$
\sup _{\|\psi\|_{L_{1}^{2}}=1}|\langle b S(\Lambda), \psi\rangle|=\sup _{\|\psi\|_{L_{1}^{2}}=1}|\langle S(\Lambda), b \psi\rangle|
$$

and also

$$
\begin{aligned}
|\langle S(\Lambda), b \psi\rangle| & \leq\|S(\Lambda)\|_{L^{2}(\partial \Omega)}\|b \psi\|_{L^{2}(\partial \Omega)} \\
& \leq\|S(\Lambda)\|_{L^{2}(\partial \Omega)}\|b\|_{L^{n-1}\|\psi\|_{L^{\frac{2(n-1)}{n-3}}(\partial \Omega)}} \\
& \leq\|S(\Lambda)\|_{L^{2}(\partial \Omega)}\|b\|_{L^{n-1}(\partial \Omega)}\|\psi\|_{L_{1}^{2}(\partial \Omega)}
\end{aligned}
$$

We now recall that for $b \in L^{\infty}(\partial \Omega)$, the operator defined by pointwise multiplication times $b$ is bounded on $L^{2}(\partial \Omega)$. Thus, for such a function $b$, $b S$ is compact on $L_{-1}^{2}(\partial \Omega)$. Since any $b \in L^{n-1}(\partial \Omega)$ can be approximated by Lipschitz functions (in the $L^{n-1}(\partial \Omega)$-norm) the estimate (4.3) yields the compactness of the operator $b S$ on $L_{-1}^{2}(\partial \Omega)$. The case $n=3$ follows from Lemma 3.1 and the fact that $\frac{1}{2} I-K^{*}$ has index 0 on $B_{2}^{-s, 2}(\partial \Omega)$ (see [8], (3.11) and (3.12) in Lemma 3.2)). 
Lemma 4.3 The operator $\mathcal{T}$ has index 0 on $L^{2}(\partial \Omega)$ for $n>3$. If $n=3$, then $\mathcal{T}$ has index 0 on $L^{2}(\partial \Omega)$ provided $b \in L^{r}(\partial \Omega)$ for some $r>2$.

This result was proved in [14], Corollary 2.4. Here we recall the main steps in the proof. Clearly, $b S=T_{b} \circ S$ is compact if $b \in L^{\infty}(\partial \Omega)$. For $n>3$, $b \in L^{n-1}(\partial \Omega)$ and $f \in L^{2}(\partial \Omega)$, we have:

$$
\|b S(f)\|_{L^{2}(\partial \Omega)} \leq\|b\|_{L^{n-1}(\partial \Omega)}\|S(f)\|_{\frac{2(n-1)}{n-3}(\partial \Omega)} .
$$

A similar argument holds for $n=3$ and $b \in L^{r}(\partial \Omega)$ for $r>2$. It follows that $b S$ can be approximated in the $L^{2}(\partial \Omega)$ operator norm by compact operators. Thus, $\mathcal{T}$ is a compact perturbation of an operator of index 0 (see Lemma 3.2, $(i))$. The proof is concluded.

Lemma 4.4 Let $0 \leq b \in L^{n-1}(\partial \Omega), b>0$ on a set of positive Surface measure. Then, if $n=3, \mathcal{T}$ is invertible on $B_{2}^{s, 2}(\partial \Omega)=L_{s}^{2}(\partial \Omega)$ for $-1<s<0$. If $n>3$, the operator $\mathcal{T}$ is invertible on $B_{2}^{s, 2}(\partial \Omega)=L_{s}^{2}(\partial \Omega)$ for $-1 \leq s \leq 0$.

Proof. We first deal with the case $n>3$. Since for $f \in L^{2}(\partial \Omega)$, one has

$$
\int_{\Omega}|\nabla \mathcal{S}(f)(X)|^{2} d x=\int_{\partial \Omega}\left(-\frac{1}{2} I+K^{*}\right)(f)(Q) S(f)(Q) d \sigma(Q),
$$

it follows at once from Lemma 3.2 and Lemma 4.3 that $\mathcal{T}$ is invertible on $L^{2}(\partial \Omega)$. Next, we show that $\mathcal{T}$ is injective on $B_{2}^{-1,2}(\partial \Omega)=L_{-1}^{2}(\partial \Omega)$. If $f \in L_{-1}^{2}(\partial \Omega)$ and $\mathcal{T}(f)=0$, then

$\left(-\frac{1}{2} I+K^{*}\right)(f)=-b S(f) \in L_{0}^{2}(\partial \Omega)=:\left\{f: f \in L^{2}(\partial \Omega)\right.$ and $\left.\int_{\partial \Omega} f d \sigma=0\right\}$.

Since $S(f) \in L^{2}(\partial \Omega)$ (see [23]), it follows from Hölder's inequality that for $p=\frac{2(n-1)}{n+1}$ one has $b S(f) \in L^{p}(\partial \Omega) \subseteq L_{-1}^{2}(\partial \Omega)$. Since $\frac{1}{2} I-K^{*}$ is an isomorphism on $L_{0}^{p}(\partial \Omega)$, there exists $g \in L_{0}^{p}(\partial \Omega)$ such that

$$
\left(\frac{1}{2} I-K^{*}\right)(g)=\left(\frac{1}{2} I-K^{*}\right)(f) .
$$

Let $f_{0} \in L^{2}(\partial \Omega) \subseteq L^{p}(\partial \Omega)$ be such that $f_{0} \in \operatorname{ker}\left(\frac{1}{2} I-K^{*}\right)$, and $\int_{\partial \Omega} f_{0} \neq 0$. Then $S\left(f_{0}\right)$ is a non zero constant on $\partial \Omega$. Assume $f_{0}$ is normalized so that $S\left(f_{0}\right)=1$. Since the index of $\frac{1}{2} I-K^{*}$ as an operator on $L_{-1}^{2}(\partial \Omega)$ is 0 , it follows that $f-g=c f_{0}$. Thus, $\mathcal{T}(g)=-c b$. But $\mathcal{T}$ is invertible on $L^{p}(\partial \Omega)$, so $g=-c f_{0}$. Hence $f=0$. It follows from Lemma 4.2 that $\mathcal{T}$ is invertible on $B_{2}^{-1,2}(\partial \Omega)$ for $n>3$. Lemma 4.4 is now obtained for $n>3$ by (complex) interpolation. 
Concerning the case $n=3$, we know from Lemma 4.2 that for $-1<s<0$ the Fredholm index of $\mathcal{T}$ on $B_{2}^{s, 2}(\partial \Omega)$ is 0 . Choosing $p=\frac{2}{1-s}<2$ it is easy to see that

$$
L^{p}(\partial \Omega) \subseteq B_{2}^{s, 2}(\partial \Omega) .
$$

If $f \in B_{2}^{s, 2}(\partial \Omega)$ is in the kernel of $\mathcal{T}$. it follows from Sobolev's Embedding Theorem that $b S(f) \in L^{p}(\partial \Omega)$, as $S(f) \in B_{2}^{s+1,2}(\partial \Omega)$. As in the case $n>3$, this implies that $\mathcal{T}$ is injective, hence invertible on $B_{2}^{s, 2}(\partial \Omega)$. Notice that, as observed in [17], the invertibility results just proved yield a number $\epsilon>0$ depending only on $b$ and the Lipschitz character of $\Omega$, such that $\mathcal{T}$ is invertible on $L^{p}(\partial \Omega)$ and on $B_{p}^{-1, p}(\partial \Omega)$ for $2-\epsilon<p<2+\epsilon$. This concludes the proof.

In view of the functional-analytic remarks recalled in the proof of Lemma 3.1, the operator $\mathcal{T}$, (which coincides with $\hat{\mathcal{T}}$ on smooth functions) is an isomorphism on $B_{1}^{-n\left(\frac{1}{p}-1\right), 1}(\partial \Omega)$ for $1-\epsilon(\Omega)<p<1$. Moreover, for $s>r$ and $s-\frac{n-1}{2} \geq r-(n-1)$, we have the embedding (see [20])

$$
B_{2}^{s 2}(\partial \Omega) \subseteq B_{1}^{r, 1}(\partial \Omega)
$$

Thus, for $t, \rho>0$ such that $0<t+\rho<1$, we have

$$
B_{\infty}^{-t \infty}(\partial \Omega) \subset B_{2}^{-t-\rho, 2}(\partial \Omega),
$$

and $\mathcal{T}$ must be injective on $B_{\infty}^{-t, \infty}(\partial \Omega)$. Since $\frac{1}{2} I-K^{*}$ has index 0 on $B_{\infty}^{-t, \infty}(\partial \Omega)$ for $t$ close to 1 (Lemma 3.2), it follows that $\mathcal{T}$ is also invertible on $B_{\infty}^{-t, \infty}(\partial \Omega)$ for $t$ close to 1 .

Next, for $\epsilon>0$, let $\mathcal{U}_{\epsilon}$ denote the plane region determined (in the $\left(s, \frac{1}{p}\right)$ plane) by the conditions $-1<s<0,0<\frac{1}{p}<1$ and

$$
\frac{1}{2}-\epsilon+\frac{\frac{1}{2}-\epsilon}{1-\epsilon} s<\frac{1}{p}<\frac{\frac{1}{2}-\epsilon}{1-\epsilon}(s+\epsilon)+1 .
$$

That is, $\mathcal{U}_{\epsilon}$ is the interior of the hexagon with vertices $(0,1),(-\epsilon, 1)$, $\left(-1, \frac{1}{2}+\epsilon\right),(-1,0),(-1+\epsilon, 0)$ and $\left(0, \frac{1}{2}-\epsilon\right)$. We are now in a position to prove our main invertibility result:

Theorem 4.5 Let $\Omega \in \mathbb{R}^{n}, n \geq 3$ be a given bounded Lipschitz domain with connected boundary $\partial \Omega, 0 \leq b \in L^{n-1}(\partial \Omega)$ with $b>0$ on a set of positive Surface measure. Then there exists a positive number $\epsilon$ depending only on $b$ and the Lipschitz character of $\Omega$ such that the operator $\mathcal{T}=-\frac{1}{2} I+K^{*}+b S$ is an isomorphism on $B_{p}^{s, p}(\partial \Omega)$ for $\left(s, \frac{1}{p}\right) \in \mathcal{U}_{\epsilon}$. If $n>3, \mathcal{T}$ is an isomorphism on $L^{q}(\partial \Omega)$ for $1<q \leq 2+\epsilon$. The region $\mathcal{U}_{\epsilon}$ is optimal, in the sense that given any $\left(s, \frac{1}{p}\right) \notin \mathcal{U}_{\epsilon}$ there exists a Lipschitz domain $\Omega_{s, p}$ such that the associated $\mathcal{T}$ is not invertible in $B_{p}^{s, p}(\partial \Omega)$. 
Proof. The proof follows from the invertibility results in Lemma 4.4 and complex interpolation (see [17]). Although interpolating the invertibility of an operator requires some care (see [6] for a counterexample), all difficulties in the present case were overcome in [8]. It was also proved there that $\mathcal{U}_{\epsilon}$ is sharp (in the above sense) for the invertibility of $-\frac{1}{2} I+K^{*}$ on $\tilde{B}_{p}^{s, p}(\partial \Omega)$ in the class of Lipschitz domains. The corresponding assertion for the invertibility of $\mathcal{T}$ follows easily from there; for example, let $\left(s, \frac{1}{p}\right) \notin \mathcal{U}_{\epsilon}$, e.g. $p \geq 2$, $-1<s<0$. If $\mathcal{T}$ were an isomorphism on $B_{p}^{s, p}(\partial \Omega)$ for such $s$ and $p$, it would follow that $\left(-\frac{1}{2} I+K^{*}\right)$ has index 0 as an operator on $B_{p}^{s, p}(\partial \Omega) \subseteq L_{-1}^{2}(\partial \Omega)$. Since the range of $-\frac{1}{2} I+K^{*}$ has codimension at least 1 , it is immediate that $\operatorname{ker}\left(-\frac{1}{2} I+K^{*}\right) \stackrel{\subseteq}{\subseteq}<f_{0}>$ (in the notation of Lemma 4.4) must have dimension one, whence $\left(-\frac{1}{2} I+K^{*}\right)$ must map $B_{p}^{s, p}(\partial \Omega)$ onto $\tilde{B}_{p}^{s, p}(\partial \Omega)$. In particular, it would follow that $-\frac{1}{2} I+K^{*}$ is an isomorphism on $\tilde{B}_{p}^{s, p}(\partial \Omega)$. But it is well known that if $\left(s, \frac{1}{p}\right) \notin \mathcal{U}_{\epsilon}$, a Lipschitz domain can be found for which $\left(-\frac{1}{2} I+K^{*}\right)$ is not an isomorphism on $\tilde{B}_{s}^{p}(\partial \Omega)$. The case $p \leq 2$ is handled similarly.

\section{The Poisson's Problem}

In this section we discuss the solution of problem (1.1). The first order of business is to properly formulate the boundary condition, which we do in Definition 5.1. We then obtain existence and a-priori estimates for the solution, which, in particular, grant uniqueness in the Sobolev class. Note that we obtain a strong solution, for which we exhibit an explicit representation as the sum of a Newtonian potential and a single layer potential.

Definition 5.1 Let $1<p<\infty, \frac{1}{p}+\frac{1}{q}=1,1-\frac{1}{p}<t<2-\frac{1}{p}, f \in L_{-t, 0}^{p}(\Omega)$, $b \in L^{n-1}(\partial \Omega)(n \geq 3)$ and $g \in B_{p}^{1-1 / p-t, p}(\partial \Omega)$. A function $u \in L_{2-t}^{p}(\Omega)$ is a solution of the boundary value problem

$$
\left\{\begin{array}{l}
\Delta u=f \quad \text { in } \Omega \\
\frac{\partial u}{\partial N}+\left.b u\right|_{\partial \Omega}=g
\end{array}\right.
$$

if for every $\phi \in B_{q}^{t-1 / q, q}(\partial \Omega)$ extended to $\tilde{\phi} \in L_{t}^{q}(\Omega)$, one has

$$
\langle\nabla u, \nabla \phi\rangle+\langle\operatorname{Tr} u, \operatorname{Tr} \phi\rangle=\langle g, \operatorname{Tr} \phi\rangle+\langle f, \phi\rangle .
$$

We observe that all pairings above make sense, on account of Lemma 2.2 and the remark thereafter, and Lemma 3.1. 
Theorem 5.2 Let $\Omega \subset \mathbb{R}^{n}, n \geq 3$ denote a bounded Lipschitz domain with connected boundary. Let $0 \leq b \in L^{n-1}, b>0$ on a set of positive Surface measure. Then there exists a positive number $\epsilon$ (depending only on $b$ and the Lipschitz character of $\Omega$ ) such that whenever $\left(t, \frac{1}{p}\right)$ belongs to the hexagon $\mathcal{V}_{\epsilon}$ with vertices $(0,1),(\epsilon, 1),\left(\frac{3}{2}-\epsilon, \frac{1}{2}+\epsilon\right),(2,0),(2-\epsilon, 0)$ and $\left(\frac{1}{2}+\epsilon, \frac{1}{2}-\epsilon\right)$, the problem (5.1) has a unique solution in $L_{2-t}^{p}(\Omega)$ for any $f$ and $g$ as specified in Definition 5.1. Letting $\mathcal{N}_{\Omega}$ denote the Newtonian potential (2.9), $\mathcal{S}$ the single layer potential (1.7) and $\mathcal{T}$ the operator (3.1), the solution is given by

$$
u(X)=\mathcal{N}_{\Omega}(f)(X)+\mathcal{S} \mathcal{T}^{-1}\left(g-\mathcal{T}\left(\mathcal{N}_{\Omega}(f)\right)\right)(X)
$$

and is subject to the a priori estimate

$$
\|u\|_{L_{2-t}^{p}(\Omega)} \leq C\left(\|f\|_{L_{-t}^{p}(\Omega)}+\|g\|_{B_{p}^{1-\frac{1}{p}-t}(\partial \Omega)}\right),
$$

where $C$ is a positive constant depending only on $p, b, t$ and the Lipschitz character of $\Omega$.

Proof. It is easy to verify that $\left(t, \frac{1}{p}\right) \in \mathcal{V}_{\epsilon}$ if and only if $\left(1-t-\frac{1}{p}, \frac{1}{p}\right) \in \mathcal{U}_{\epsilon}$, (see (4.4)). The existence and representation statements follow then immediately from Theorem 2.3, by reducing (5.1) to a homogeneous problem with non-zero boundary data in $B_{p}^{1-t-1 / p, p}(\partial \Omega)$, combined with the invertibility results in the previous section, from which a solution of the form (1.4) is obtained. Indeed, recall that

$$
\Delta \mathcal{N}_{\Omega}(f)=f .
$$

From Theorem 2.3 and Theorem 4.5, we have that

$$
h=\mathcal{T}^{-1}\left(\frac{\partial \mathcal{N}_{\Omega}}{\partial N}+b \operatorname{Tr} \mathcal{N}_{\Omega}(f)\right)
$$

is well defined as an element of $B_{p}^{1-t-\frac{1}{p}, p}(\partial \Omega)$. In light of formula (1.14) and Theorem 2.4, it is now clear that

$$
\mathcal{S}\left(\mathcal{T}^{-1}(g)-h\right)(X) .
$$

is a solution to problem 5.1 with $f=0$ and boundary datum $g-\mathcal{T}(h)$ (see (1.4)). Thus, we obtain the representation 5.3. It remains to prove uniqueness. For $\left(t, \frac{1}{p}\right) \in \mathcal{V}_{\epsilon}$, let $u \in L_{2-t}^{p}(\Omega)$ be a solution of (5.1) with $f=0$ and $g=0$. Then, $\operatorname{Tr}(u) \in B_{p}^{2-1 / p-t, p}(\partial \Omega)$. Let now $w \in L_{t}^{q}(\Omega)$ stand for a solution to (5.1) with $f=0$ and an arbitrary boundary data $g \in B_{q}^{1 / p+t-2, q}(\partial \Omega)$. It can be readily concluded from (5.2) that $\operatorname{Tr} u \in$ ker $g$. Uniqueness now follows from the corresponding result for the Dirichlet problem with boundary data in $B_{p}^{2-1 / p-t, p}(\partial \Omega)$ (see [11]). 


\section{The 3-dimensional Lamé System}

As a further application of the functional-analytic ideas outlined in the previous chapters, we present here a version of the Poisson's Problem for the 3-dimensional Lamé System of elastostatics. The dimensional restriction is due to the fact that the endpoint result corresponding to $F_{1}^{0,2}$ is only known for $n=3$ (see [3]). In this Section, the terminology associated with the domain $\Omega$ has the same meaning as before and 3-dimensional vector valued functions on $\Omega$ will be denoted by boldface. To simplify the notations, we continue to use $A_{p}^{s, q}$ for the space of vector-valued functions with components in $A_{p}^{s, q}$. For $\mu>0$ and $\lambda>-\frac{2}{3} \mu$ (called the Lamé constants), the Lamé operator is defined as

$$
\mathcal{L}:=\mu \Delta \mathbf{u}+(\lambda+\mu) \nabla \operatorname{div} \mathbf{u} .
$$

Of particular interest in applications is the (Neumann-type) Traction boundary condition, namely

$$
\frac{\partial \mathbf{u}}{\partial \nu}:=\left(\lambda \operatorname{div} \mathbf{u} N+\mu\left[\nabla \mathbf{u}+\nabla^{t} \mathbf{u}\right] N\right)
$$

where the superscript $t$ indicates the usual transposition of matrices. We state Lemma 6.1, whose proof can be obtained by slightly modifying the arguments in Lemma 3.1 for $n=3$.

Lemma 6.1 Let $b \in L^{2}(\partial \Omega)$ be a scalar function, $1<p<\infty$ and $0<s<1$. Then the map $T_{b}$, defined on smooth vector fields as the pointwise product times $b$ (componentwise), maps $B_{p}^{1-s, p}(\partial \Omega)$ boundedly into $B_{p}^{-s, p}(\partial \Omega)$.

Next, we let $\mathbf{g} \in B_{p}^{1-\frac{1}{p}-t, p}(\partial \Omega), 1<p<\infty, \frac{1}{p}+\frac{1}{q}=1$ and $\frac{1}{q}<t<1+\frac{1}{q}$. By a solution to the Poisson's problem

$$
\left\{\begin{array}{l}
\mathcal{L} \mathbf{u}=\mathbf{f} \in L_{-t, 0}^{p}(\Omega) \quad \text { in } \Omega \\
\frac{\partial \mathbf{u}}{\partial \nu}+\left.b \mathbf{u}\right|_{\partial \Omega}=\mathbf{g}
\end{array} .\right.
$$

we mean a vector-valued function $\mathbf{u} \in L_{2-t}^{q}(\Omega)$ such that for every $\boldsymbol{\psi} \in$ $B_{q}^{t-1 / q, q}(\partial \Omega)$ extended to $\tilde{\boldsymbol{\psi}} \in L_{t}^{q}(\Omega)$, the equality $\langle\mathbf{f}, \tilde{\boldsymbol{\psi}}\rangle+\mu\left\langle\nabla \mathbf{u}+(\nabla \mathbf{u})^{t}, \nabla \tilde{\boldsymbol{\psi}}+(\nabla \tilde{\boldsymbol{\psi}})^{t}\right\rangle+\lambda\langle\operatorname{div} \mathbf{u}, \operatorname{div} \tilde{\boldsymbol{\psi}}\rangle+\langle b \mathbf{u}, \boldsymbol{\psi}\rangle=\langle\mathbf{g}, \boldsymbol{\psi}\rangle$

holds. We point out that the above expression is well defined on account of Lemma 6.1 and (a suitable version of) Lemma 2.2. The matrix of fundamental solutions for the 3 -dimensional system of elastostatics is given by $\boldsymbol{\Gamma}(X)=\left(\Gamma_{i j}\right)_{i j}(X), X \in \mathbb{R}^{3} \backslash\{0\}$, with

$$
\Gamma_{i j}(X)=\frac{1}{8 \pi}\left(\frac{1}{\mu}+\frac{1}{2 \mu+\lambda}\right) \delta_{i j}+\frac{1}{8 \pi}\left(\frac{1}{\mu}-\frac{1}{2 \mu+\lambda}\right) \frac{X_{i} X_{j}}{|X|^{3}}
$$


In analogy with the terminology in Section 2, we define the Elastic Potential of a density $\mathbf{f}, \mathcal{N}_{\Omega}(\mathbf{f})$ via the analogue of formula (2.9), where $\Gamma$ stands for the matrix with entries given by (6.2). The Elastic Potential bears mapping properties analogous to those of the Newtonian potential in Theorem 2.3. This can be proved by carrying out the minor obvious modifications to fit the present setting. We also define the single, double and boundary layer potential operators with density $\mathbf{f}$ respectively as

$$
\mathcal{S}(\mathbf{f})(X)=\langle\mathbf{f}, \Gamma(X-\cdot)\rangle, X \in \mathbb{R}^{3},
$$

for $\mathbf{f} \in \mathcal{L}^{\prime}(\partial \Omega)$, and, for

$$
\mathbf{f}: \partial \Omega \rightarrow \mathbb{C},
$$

we let

$$
K(\mathbf{f})(P)=p \cdot v \cdot \int_{\partial \Omega}\left(\frac{\partial}{\partial \nu(Q)} \Gamma(P-\cdot)\right) \mathbf{f}(Q) d \sigma(Q)
$$

and

$$
\mathcal{D}(\mathbf{f})(X)=\int_{\partial \Omega}\left(\frac{\partial}{\partial \nu(Q)} \Gamma(X-\cdot)\right) \mathbf{f}(Q) d \sigma(Q), X \in \mathbb{R}^{3} \backslash \partial \Omega .
$$

The corresponding boundary traces are given by

$$
\begin{aligned}
\lim _{X \rightarrow P, X \in \pm \gamma(P)} \frac{\partial}{\partial \nu} \mathcal{S}(\mathbf{f})(X)= \\
=\mp \frac{1}{2} \mathbf{f}(P)+p \cdot v \cdot \int_{\partial \Omega}\left(\frac{\partial}{\partial \nu(P)} \Gamma(P-\cdot)\right)^{t} \mathbf{f}(Q) d \sigma(Q) \\
=\mp \frac{1}{2} f(P)+K^{*}(\mathbf{f})(P)
\end{aligned}
$$

and

$$
\begin{aligned}
\lim _{X \rightarrow P, X \in \pm \gamma(P)} \mathcal{D}(\mathbf{f})(X) \\
= \pm \frac{1}{2} \mathbf{f}(P)+p \cdot v \cdot \int_{\partial \Omega}\left(\frac{\partial}{\partial \nu(Q)} \Gamma(P-\cdot)\right) \mathbf{f}(Q) d \sigma(Q) \\
= \pm \frac{1}{2} f(P)+K(\mathbf{f})(P) .
\end{aligned}
$$

In (6.5) and (6.6), the conormal derivative $\frac{\partial}{\partial \nu}$ is applied to every column vector of $\Gamma$. As in (1.13) and (1.14), if $f \in B_{p}^{s, p}(\partial \Omega)$ formulas (6.5) are to be interpreted in the trace sense. 
Furthermore, for $1 \leq p \leq \infty, 0<s<1$ and density $\mathbf{f} \in B_{p}^{-s, p}(\partial \Omega)$, the identity (6.5) is to be interpreted in the following weak sense: for any $\boldsymbol{\psi} \in B_{q}^{s, q}(\partial \Omega)$ extended to $\tilde{\boldsymbol{\psi}} \in L_{s+1 / q}^{q}(\Omega)$, the equality

$$
\mu\left\langle\nabla \mathbf{u}+(\nabla \mathbf{u})^{t}, \nabla \tilde{\boldsymbol{\psi}}+(\nabla \tilde{\boldsymbol{\psi}})^{t}\right\rangle+\lambda\langle\operatorname{div} \mathbf{u}, \operatorname{div} \tilde{\boldsymbol{\psi}}\rangle=\left\langle\left(-\frac{1}{2} I+K^{*}\right) \mathbf{f}(P), \boldsymbol{\phi}\right\rangle
$$

holds.

Next we state the analogue of Theorem 2.4, namely:

Theorem 6.2 For $0<s<1$ and $1<p<\infty$, the elastic single layer potential

$$
\mathcal{S}: B_{p}^{-s, p}(\partial \Omega) \rightarrow B_{p}^{1-s+\frac{1}{p}, p}(\Omega) \cap L_{1-s+\frac{1}{p}, p}^{p}(\Omega)
$$

and the elastic double layer potential

$$
\mathcal{D}: B_{p}^{s, p}(\partial \Omega) \rightarrow B_{p}^{s+\frac{1}{p}, p}(\Omega) \cap L_{s+\frac{1}{p}}^{p}(\Omega)
$$

are bounded linear operators. Here, the intersection of two Banach spaces $X$ and $Y$ is endowed with the norm

$$
\|f\|_{X \cap Y}=\max \left\{\|f\|_{X},\|f\|_{Y}\right\} .
$$

Let $\Psi$ be the (six-dimensional) vector space of $\mathbb{R}^{3}$-valued functions $\boldsymbol{\psi}=\left(\psi_{j}\right)_{j}$ defined on $\Omega$ and satisfying

$$
\partial_{i} \boldsymbol{\psi}_{j}+\partial_{j} \boldsymbol{\psi}_{i}
$$

and for any space $\chi(\partial \Omega)$ of distributions on $\partial \Omega$, let

$$
\chi_{\Psi}(\partial \Omega)=\{\Lambda \in \chi(\partial \Omega):\langle\Lambda, \boldsymbol{\psi}\rangle=0 \forall \boldsymbol{\psi} \in \chi(\partial \Omega)\} .
$$

The following is the analogue of Lemma 3.2, with $K$ now defined by (6.4) :

Lemma 6.3 The operator $\frac{1}{2} I-K$ is Fredholm and has index 0 as an operator on $L^{2}(\partial \Omega)$; on $L_{1}^{2}(\partial \Omega)$, and on $B_{1}^{1-s, 1}(\partial \Omega)$, for sufficiently small positive s. Moreover, $\frac{1}{2} I-K^{*}$ is Fredholm and has index 0 as an operator on $F_{1}^{0,2}(\partial \Omega)$.

Proof. It was shown in [4] that $\frac{1}{2} I-K^{*}$ (and hence $\frac{1}{2} I-K$ ) is Fredholm and has index 0 on $L^{2}(\partial \Omega)$. The kernel of $\frac{1}{2} I-K^{*}$ as an operator on $L^{2}(\partial \Omega)$ is precisely the space of restrictions to $\partial \Omega$ of functions in $\Psi$ (see above), which from now on we denote $\Psi(\partial \Omega)$. Proceeding as in Lemma 3.2, one easily concludes that

$$
L^{2}(\partial \Omega)=\Psi(\partial \Omega) \oplus \operatorname{range}\left(\frac{1}{2} I-K^{*}\right)=\operatorname{range}\left(\frac{1}{2} I-K\right) \oplus \Psi(\partial \Omega) .
$$


The argument presented in Lemma 3.2 to show the closedness of range $\left(\frac{1}{2} I-K\right)$ (as an operator on $L_{1}^{2}(\partial \Omega)$ ) now follows along the same lines once it is realized that for a $3 \times 3$ matrix $B$ and a column vector function $\mathbf{u}$, we have

$$
\int_{\Omega}(\mathcal{L}(B \mathbf{u})-B \mathcal{L} \mathbf{u})=\int_{\partial \Omega}\left(\frac{\partial B}{\partial \nu} \mathbf{u}-B \frac{\partial \mathbf{u}}{\partial \nu}\right) d \sigma .
$$

Now the range of $\frac{1}{2} I-K$ as an operator acting on $L_{1}^{2}(\partial \Omega)$ consists of those functions in $L_{1}^{2}(\partial \Omega)$ whose integral against elements of $\Psi(\partial \Omega)$ vanishes. This can be proved via (6.7) and the aforementioned integration by parts. Also, $\operatorname{ker}\left(\frac{1}{2} I-K\right)$ is $\Psi(\partial \Omega)=\left(L_{\Psi}^{2}(\partial \Omega)\right)^{\perp}$. Therefore, $\frac{1}{2} I-K^{*}$ is Fredholm and has index 0 on $L_{-1}^{2}(\partial \Omega)$. The results in [3] allow us to show (via the methods in Theorem 3.2) that $\frac{1}{2} I-K^{*}$ and $\frac{1}{2} I-K$ are Fredholm and have index 0 both on $F_{1}^{0,2}(\partial \Omega)$ and on $B_{1}^{1-s, 1}(\partial \Omega)$ (for small positive $s$ ). By duality, and since $\operatorname{ker}\left(\frac{1}{2} I-K^{*}\right)=\Psi$ on $B_{\infty}^{s-1, \infty}(\partial \Omega)$ (note that $B_{\infty}^{s-1, \infty}(\partial \Omega) \subseteq L_{-1}^{2}(\partial \Omega$ ) and that all functions in $\Psi$ are smooth), it follows at once that the index of $\left(\frac{1}{2} I-K^{*}\right)$ as an operator on $B_{\infty}^{s-1, \infty}(\partial \Omega)$ is zero, which finishes the proof.

We recall from the $H^{1}$ theory established in [3] that

$$
S: F_{1}^{0,2}(\partial \Omega) \rightarrow F_{1}^{1,2}(\partial \Omega)
$$

is an isomorphism, and that if $\mathbf{f} \in F_{1}^{0,2}(\partial \Omega)$, then the non-tangential maximal function $M(S(\mathbf{f}))$ is in $L^{1}(\partial \Omega)$

Theorem 6.4 Let $\Omega \subset \mathbb{R}^{3}$ be a bounded Lipschitz domain with connected boundary $\partial \Omega, b \in L^{2}(\partial \Omega), b \geq 0, b>0$ on a set of positive Surface measure. There exists a positive number $\epsilon$ depending only on the Robin coefficient $b$ and $\Omega$ such that the operator $\mathcal{T}=-\frac{1}{2} I+K^{*}+b \mathcal{S}$ is invertible on $B_{p}^{s, p}(\partial \Omega)$ when the point $\left(s, \frac{1}{p}\right)$ lies in the interior of the hexagon $\mathcal{U}_{\epsilon}$ with vertices $(-\epsilon, 1),(0,1),\left(0, \frac{1}{2}-\epsilon\right),(-1+\epsilon, 0),(-1,0)$ and $\left(-1, \frac{1}{2}+\epsilon\right)$. Moreover, the hexagon $\mathcal{U}_{\epsilon}$ is optimal in the sense described in Theorem 4.5.

Proof. The proof goes along the same lines as in the scalar case for $n=3$. We know from Lemma 3.2 that $\frac{1}{2} I-K^{*}$ is Fredholm and has index 0 as an operator on $B_{2}^{s, 2}(\partial \Omega)$ for $-1 \leq s \leq 0$. Using Lemma 6.1 we conclude that for $b \in L^{2}(\partial \Omega), 1<p<\infty$ and $0<s<1$, the operator

$$
b S: B_{p}^{s-1, p}(\partial \Omega) \rightarrow B_{p}^{s-1, p}(\partial \Omega)
$$

is compact. Next, we observe that for $-1<s<0, L^{2}(\partial \Omega) \subseteq B_{2}^{s, 2}(\partial \Omega) \subseteq$ $B_{2}^{-1,2}(\partial \Omega)$; an argument similar to the proof of Lemma 4.4 shows now that as an operator on $B_{2}^{s, 2}(\partial \Omega)$, $\operatorname{ker} \mathcal{T}=\{0\}$. Hence, $\mathcal{T}$ is an isomorphism on $B_{2}^{s, 2}(\partial \Omega)$ for $-1<s<0$. Writing

$$
\mathbf{u}(X)=\mathcal{S}(\mathbf{f})(X)
$$


for $\mathbf{f} \in F_{1}^{0,2}(\partial \Omega)$, the identity (see [4])

$$
\int_{\partial \Omega} \mathbf{u} \frac{\partial \mathbf{u}}{\partial \nu} d \sigma=\int_{\Omega}\left(\lambda(\operatorname{div} \mathbf{u})^{2}+\frac{\mu}{2}\left|\nabla \mathbf{u}+(\nabla \mathbf{u})^{t}\right|^{2}\right) d X
$$

(which is proved via integration by parts) allows then to proceed along the same lines as in Lemma 4.1 and obtain that $-\frac{1}{2} I+K^{*}+b S$ is invertible on $F_{1}^{0,2}(\partial \Omega)$. Proceeding now as in the remarks following the proof of Lemma 4.4, one concludes that for small positive $s$, the operators

$$
\mathcal{T}: B_{1}^{-s, 1}(\partial \Omega) \rightarrow B_{1}^{-s, 1}(\partial \Omega)
$$

and

$$
\mathcal{T}: B_{\infty}^{s-1, \infty}(\partial \Omega) \rightarrow B_{\infty}^{s-1, \infty}(\partial \Omega)
$$

are isomorphisms. The counterexamples presented in [17] in conjunction with an argument similar to the one presented in the proof of Theorem 4.5 yield the optimality claim.

To conclude, we observe that as in Section 5, Theorem 6.4 yields:

Theorem 6.5 Let $\Omega \subset \mathbb{R}^{3}$ denote a bounded Lipschitz domain with connected boundary, $b$ as in Theorem 6.4. Then there exists a positive number $\epsilon$, which depends only on $b$ and the Lipschitz character of $\Omega$ such that whenever $\left(t, \frac{1}{p}\right)$ belongs to the hexagon $\mathcal{V}_{\epsilon}$ described in Theorem 5.2, Problem (1.2) has a unique solution $\mathbf{u}(X)$ in $L_{2-t}^{p}(\Omega)$, which is given by

$$
\mathbf{u}(x)=\mathcal{N}_{\Omega}(\mathbf{f})(X)+\mathcal{S} \mathcal{T}^{-1}\left(\mathbf{g}-\mathcal{T}\left(\mathcal{N}_{\Omega}\right)(\mathbf{f})\right)(X),
$$

and is subject to the following a priori estimate:

$$
\|\mathbf{u}\|_{L_{2-t}^{p}(\Omega)} \leq C\left(\|\mathbf{f}\|_{L_{-t}^{p}(\Omega)}+\|\mathbf{g}\|_{B_{p}^{1-\frac{1}{p}-t, p}(\partial \Omega)}\right) .
$$

Here, $C$ is a positive constant, which depends only on $p, b, t$ and the Lipschitz character of $\Omega$.

\section{References}

[1] Coifman, R., McIntosh, A. and Meyer, Y.: L'intégrale de Cauchy définit un opérateur borné sur $L^{2}$ pour les courbes Lipschitziennes. Ann. of Math. (2) 116 (1982), 361-387.

[2] Dahlberg, B. E. J. And Kenig, C. E.: Hardy spaces and the Neumann problem in $L^{p}$ for Laplace's equation in Lipschitz domains. Ann. of Math. (2) 125 (1987), 437-465. 
[3] Dahlberg, B. E. J. And Kenig, C. E.: $L^{p}$ estimates for the threedimensional systems of elastostatics on Lipschitz domains. In Analysis and partial differential equations, 621-634. Lecture Notes in Pure and Applied Math. 122, Dekker, New York, 1990.

[4] Dahlberg, B. E. J., Kenig, C. E. and Verchota, G. C.: Boundary value problems for the systems of elastostatics in Lipschitz domains. Duke Math. J. 57 (1988), 795-818.

[5] Edmunds, D. E. And Triebel, H.: Function spaces, entropy numbers, differential operators. Cambridge Tracts in Mathematics 120. Cambridge University Press, Heidelberg, 1996.

[6] Fabes, E. B., Jodeit, M. JR. And Lewis, J. E.: On the spectra of a Hardy kernel. J. Funct. Anal. 21 (1976), 187-194.

[7] Fabes, E. B., Jodeit, M. JR. And Rivière, N. M.: Potential techniques for boundary value problems on $C^{1}$-domains. Acta Math. 141 (1978), $165-186$.

[8] Fabes, E., Méndez, O. and Mitrea, M.: Boundary layers on SobolevBesov spaces and Poisson's equation for the Laplacian in Lipschitz domains. J. Funct. Anal. 159 (1998), 323-368.

[9] Frazier, M. And Jawerth, B.: A discrete transform and decompositions of distribution spaces. J. Funct. Anal. 93 (1990), 34-170.

[10] Grisvard, P.: Elliptic problems in nonsmooth domains. Monographs and Studies in Mathematics 24. Pitman, Boston, 1985.

[11] Jerison, D. and Kenig, C.: The inhomogeneous Dirichlet problem in Lipschitz domains. J. Funct. Anal. 130 (1995), 161-219.

[12] Jonsson, A. And Wallin, H.: Function Spaces on subsets of $\mathbb{R}^{n}$. Math. Rep. 2. Harwood Academic, New York, 1984.

[13] Kenig, C.: Harmonic analysis techniques for second order elliptic boundary value problems. CBMS Regional Conference Series in Mathematics 83. American Mathematical Society, Providence, RI, 1994.

[14] Lanzani, L. And Shen, Z.: On the Robin boundary condition for Laplace's equation in Lipschitz domains. Comm. Partial Differential Equations 29 (2004), 91-109.

[15] Lions, J.-L. And Magenes, E.: Non-Homogeneous boundary value problems and applications, I, II. Die Grundlehren der mathematischen Wissenschaften 181, 182. Springer Verlag, New York-Heidelberg, 1972.

[16] Méndez, O. And Mitrea, M.: Complex powers of the Neumann Laplacian in Lipschitz domains. Math. Nachr. 223 (2001), 77-88.

[17] Méndez, O. And Mitrea, M.: The Banach envelopes of Besov and Triebel-Lizorkin spaces and applications to partial differential equations. J. Fourier Anal. Appl. 6 (2000), 503-531. 
[18] Mitrea, M. and Taylor, M.: Potential theory on Lipschitz domains in Riemannian manifolds: the case of Dini metric tensors. Trans. Amer. Math. Soc. 355 (2003), no. 5, 1961-1985.

[19] Runst, T. And Sickel, W.: Sobolev spaces of fractional order, Nemytskij operators, and nonlinear partial differential equations. De Gruyter Series in Nonlinear Analysis and Applications 3. Walter de Gruyter, Berlin, 1996.

[20] Triebel, H.: Theory of function spaces. Monographs in Mathematics 78. Birkhäuser Verlag, Basel, 1983.

[21] Triebel, H.: Theory of function spaces II. Monographs in Mathematics 84. Birkhäuser Verlag, Basel, 1992.

[22] Triebel, H.: Interpolation theory, function spaces, differential operators. Second edition. Johann Ambrosius Barth, Heidelberg, 1995.

[23] Verchota, G.: Layer potentials and regularity for the Dirichlet problem for Laplace's equation in Lipschitz domains. J. Funct. Anal. 59 (1984), $572-611$.

Recibido: 11 de septiembre de 2003

Loredana Lanzani

Department of Mathematical Sciences

University of Arkansas

538 Holtz Hall

Fayetteville, AR, 72701 USA

lanzani@caccioppoli. uark.edu

Osvaldo Méndez

Department of Mathematics

University of Texas at El Paso

500W University Ave., 124 Bell Hall

El Paso, TX, 79968, USA

mendez@math . utep.edu

http://www.math.utep.edu/Faculty/mendez

The first author is supported in part by NSF grant No. DMS-9800794. 